\title{
Pomegranate (Punica granatum) juice reduces oxidative injury and improves sperm concentration in a rat model of testicular torsion-detorsion
}

\author{
DOGAN ATILGAN $^{1}$, BEKIR PARLAKTAS ${ }^{1}$, NIHAT ULUOCAK ${ }^{1}$, YUSUF GENCTEN $^{1}$, \\ FIKRET ERDEMIR $^{1}$, HUSEYIN OZYURT $^{2}$, UNAL ERKORKMAZ ${ }^{3}$ and HUSEYIN ASLAN ${ }^{4}$ \\ Departments of ${ }^{1}$ Urology and ${ }^{2}$ Biochemistry, Faculty of Medicine, Gaziosmanpasa University, Tokat 60100; \\ ${ }^{3}$ Department of Biostatistics and Medical Informatics, Faculty of Medicine, Sakarya University, \\ Sakarya 54100; ${ }^{4}$ Department of Histology and Embryology, Faculty of Medicine, \\ Gaziosmanpasa University, Tokat 60100, Turkey
}

Received January 22, 2014; Accepted June 3, 2014

DOI: $10.3892 / e t m .2014 .1782$

\begin{abstract}
The present study aimed to evaluate the effect of pomegranate juice (PJ) on oxidative stress (OS) and sperm concentration in a rat model of testicular torsion-detorsion. A total of 21 Wistar albino rats were randomly divided into three groups, each consisting of seven rats, as follows: i) control group, which underwent sham surgery; ii) ischemia/reperfusion (I/R) group, designed to determine the effects of the testicular torsion-detorsion process on rats; and iii) $\mathrm{PJ}+\mathrm{I} / \mathrm{R}$ group, designed to evaluate the effect of $\mathrm{PJ}$ on the OS and sperm cell concentrations induced by the torsion-detorsion process. In the $\mathrm{PJ}+\mathrm{I} / \mathrm{R}$ group, the rats were given $0.4 \mathrm{ml} /$ day $\mathrm{PJ}$ orally over a period of eight weeks prior to surgery. Ipsilateral orchiectomy was carried out and $5-\mathrm{cm}^{3}$ blood samples were obtained from the inferior vena cava of all rats. Biochemical analyses were performed to calculate the superoxide dismutase (SOD) activity and malondialdehyde (MDA) levels in the testicular tissue and serum. The concentrations of spermatids, spermatocytes and spermatogonia in the seminiferous tubules were assessed using histopathological methods. Serum and tissue SOD and MDA levels were significantly higher in rats from the I/R group compared with the control group $(\mathrm{P}<0.001)$. PJ treatment significantly decreased the SOD and MDA levels in both the serum and testicular tissue of the rats $(\mathrm{P}<0.001)$. The spermatid, spermatocyte and spermatogonia concentrations were significantly reduced in the $\mathrm{I} / \mathrm{R}$ group compared with the control group $(\mathrm{P}<0.001)$. $\mathrm{PJ}$ treatment significantly improved the concentrations of
\end{abstract}

Correspondence to: Dr Dogan Atilgan, Department of Urology, Faculty of Medicine, Gaziosmanpasa University, Muhittin Fisunoglu Street, Tokat 60100, Turkey

E-mail: datilgan@msn.com

Key words: testis, torsion, ischemia reperfusion, oxidative stress, pomegranate juice spermatids, spermatocytes and spermatogonia compared with those in the $\mathrm{I} / \mathrm{R}$ group $(\mathrm{P}=0.008)$. The experimentally established testicular torsion-detorsion model led to OS in the rat testes. Daily consumption of PJ prior to surgery reduced OS parameters and improved sperm cell concentrations.

\section{Introduction}

The pomegranate (Punica granatum) is a small, fruit-bearing tree that has been cultivated and naturalized since ancient times throughout the entire Mediterranean region. One of its extracts, pomegranate juice (PJ), is consumed around the world. A previous study revealed that PJ contains certain constituents that appear to have beneficial therapeutic properties, including antioxidative effects (1). Consequently, the protective effect of PJ consumption on various diseases is receiving considerable attention at present in all medical fields. The anticancer, antimicrobial, antiproliferative and cardioprotective effects of PJ and its metabolites have also been demonstrated in numerous previous studies (2-5). Pomegranate contains a number of polyphenols, including anthocyanins, minor flavonoids and punicalagin, which is the most important member of the ellagitannins family. The punicalagin is the largest polyphenol among the pomegranate ellagitannins and it is responsible from most of the antioxidant activity of the PJ (6). The potent antioxidant effects of polyphenols have been demonstrated in clinical and experimental studies $(7,8)$. Thus, the pomegranate and its extracts have powerful antioxidant effects, which have also been revealed for other fruit juices, including grape, blackberry and branberry juice, and in green tea (9).

Testicular torsion is a rarely observed, serious urologic emergency, usually encountered in prepubertal males, which requires immediate surgical examination for proper treatment. It has been hypothesized that treatment for testicular torsion may lead to long-term infertility due to ischemia/ reperfusion $(I / R)$ injury which occurs following surgical correction of the torsed testis (10). Oxidative stress (OS) is a complex phenomenon that causes tissue damage and occurs 
due to an excessive production of reactive oxygen species (ROS) that are not adequately eliminated by the natural antioxidant defence mechanisms in the body. OS may cause significant damage to tissues by inducing differentiation in the cell membranes leading to irreversible cellular damage (11). In this context, OS increases the levels of malondialdehyde (MDA) and protein carbonyls (PCs) in the tissues, which are the end products of lipid peroxidation and protein oxidation, respectively.

Under physiological conditions, the enzymes belonging to the natural antioxidant defence system, including superoxide dismutase (SOD), catalase and glutathione peroxidase (GSH-Px), eliminate the ROS, which are released as a result of normal cellular metabolism (12). Excess levels of ROS particularly target unsaturated fatty acids, which play an important role in the constitution of cell membranes. Since the membranes of sperm contain a high abundance of unsaturated fatty acids (13), these cells are severely affected by peroxidation damage caused by ROS. Lipid peroxidation destroys the structure of the lipid matrix in the membranes of spermatozoa and is associated with the loss of motility and defects in membrane integrity (14).

In the present experimental study, a rat model of testicular torsion-induced OS was established. The biochemical and histopathological effects of daily PJ consumption on the OS parameters and sperm concentrations of the rats were subsequently evaluated.

\section{Materials and methods}

Study animals. Upon obtaining consent from the Gaziosmanpasa University Local Ethics Committee for Animal Experiments (Tokat, Turkey), 21 male Wistar albino rats (aged 5-6 months) were purchased Gaziosmanpasa University Experimental Medicine Research Center (Tokat, Turkey) for use in the present study. The experimental animals were housed at $18-22^{\circ} \mathrm{C}$, under a $12 \mathrm{~h}$ light/dark cycle throughout the study period. Surgical procedures were performed under ketamine/xylazine anesthesia in sterile conditions. All rats were sacrificed following the experimental procedures.

Group allocation. The rats were randomly divided into three groups, each group consisting of seven rats, as follows: i) control group, which underwent a sham surgical procedure to determine the basal values for biochemical and histopathological evaluations; ii) I/R group, designed to study the effects of the testicular torsion-detorsion process on the ipsilateral testicle and; iii) $\mathrm{PJ}+\mathrm{I} / \mathrm{R}$ group, designed to determine the effect of PJ on OS indicators and sperm parameters following unilateral testicular torsion-detorsion. In the $\mathrm{PJ}+\mathrm{I} / \mathrm{R}$ group, the rats were given $0.4 \mathrm{ml} /$ day commercially-available PJ (Ersu ${ }^{\circledR}$ Fruit and Food Industries Inc., Konya, Turkey) orally over a period of eight weeks prior to surgery.

Surgical procedure. In the control group rats, the testicle was exposed through a midline incision, a 4-0 silk suture was fixed through the tunica albuginea and the testicle was replaced into the scrotum with no additional intervention. In the rats in the $I / R$ and $P J+I / R$ groups, the tunica vaginalis was opened and the right testis exposed to allow surgical intervention. It was rotated $720^{\circ}$ in a clockwise direction and maintained in this torsed position by fixing the testicle to the scrotum with a 4-0 silk suture. After $1 \mathrm{~h}$, the spermatic cord was detorsed and orchiectomy was performed on the right side. The resected testicular tissue specimens were separated into two parts for histopathological (sperm parameters) and biochemical (tissue MDA and SOD level) analyses. Furthermore, $\sim 5-\mathrm{cm}^{3}$ blood samples were obtained from the inferior vena cava of the rats to determine the blood levels of MDA and SOD.

Biochemical analyses. Blood samples were drawn into heparin-free tubes for biochemical analysis. Following centrifugation $\left(2,000 \mathrm{x}\right.$ for $15 \mathrm{~min}$ at $\left.4^{\circ} \mathrm{C}\right)$, the serum samples were frozen and stored at $-70^{\circ} \mathrm{C}$ until required. Commercially-available chemical supplies (Sigma; St. Louis, MO, USA) were used for the determination of the following parameters in the serum samples.

Serum antioxidant enzyme analysis. Total ( $\mathrm{Cu}-\mathrm{Zn}$ and $\mathrm{Mn})$ SOD [enzyme commission (EC) number 1.15.1.1) activity was determined according to a previous method described by Sun et al (15). The principle of the method is based on the inhibition of nitroblue tetrazolium (NBT) reduction by the xanthine-xanthine oxidase system as a superoxide generator. Activity was assessed in the ethanol phase of the supernatant following the addition of $1.0 \mathrm{ml}$ ethanol/chloroform mixture $(5: 3, v / v)$ to the same volume of sample and centrifugation $(1,500 \times \mathrm{g}$ for $10 \mathrm{~min})$. One unit of SOD was defined as the amount causing a $50 \%$ inhibition of the NBT reduction rate. The SOD activity was expressed in $\mathrm{U} / \mathrm{ml}$.

Determination of the levels of MDA. The level of tissue thiobarbituric acid reactive substance (TBARS) was determined by a method based on a reaction with thiobarbituric acid (TBA) at $90-100^{\circ} \mathrm{C}$ that was previously described by Esterbauer and Cheeseman (16). In the TBA test reaction, MDA or MDA-like substances and TBA react to produce a pink pigment with an absorption maximum at $532 \mathrm{~nm}$. The reaction was performed at $\mathrm{pH} 2-3$ and $90^{\circ} \mathrm{C}$ for $15 \mathrm{~min}$. The sample was mixed with a double volume of cold $10 \%(\mathrm{w} / \mathrm{v})$ trichloroacetic acid to precipitate the protein. The precipitate was pelleted by centrifugation (1,500 x g for $10 \mathrm{~min}$ ) and an aliquot of the supernatant was reacted with an equal volume of $0.67 \%(\mathrm{w} / \mathrm{v}) \mathrm{TBA}$ in a boiling water bath for $10 \mathrm{~min}$. Following cooling, the absorbance at $532 \mathrm{~nm}$ was measured (GBC Cintra 10e UV/VIS Spectrophotometer, Victoria, Australia). Results are expressed in $\mathrm{ng} / \mathrm{ml}$, according to the graphic standard prepared from measurements with a standard solution (1,1,3,3-tetramethoxypropane).

Histological examination. The rat testes were fixed in Bouin's solution (Polysciences Europe GmbH, Eppelheim, BadenWürttemberg, Germany) and passed through an increasing alcohol series. The testicular tissue was processed for paraffin embedding following treatment with xylene and paraffin. Subsequently, 5- and $20-\mu$ m-thick sections were obtained with a rotary microtome (LeicaRM 2135, Leica Instruments, Nussloch, Germany). The sections were randomly sampled and stained with Periodic acid-Schiff (PAS). 
Table I. Levels of superoxide dismutase (SOD) and malondialdehyde (MDA) in the serum and testicular tissue of rats from the different groups.

\begin{tabular}{lcccr}
\hline Variable & Control & I/R & PJ+I/R & P-value \\
\hline tSOD $(\mathrm{U} / \mathrm{ml})$ & $13.18(7.86-17.54)$ & $29.37(26.45-38.26)^{\mathrm{a}}$ & $12.04(8.61-13.66)$ & $<0.001$ \\
tMDA $(\mathrm{ng} / \mathrm{ml})$ & $86.09(66-139.71)$ & $270.37(237.86-316.39)^{\mathrm{a}}$ & $81.84(70.08-103.53)$ & $<0.001$ \\
sSOD $(\mathrm{U} / \mathrm{ml})$ & $26.76(23.67-29.52)$ & $73.45(61.66-81.47)^{\mathrm{a}}$ & $22.1(16.9-25.2)$ & $<0.001$ \\
sMDA $(\mathrm{ng} / \mathrm{ml})$ & $1660.6(1589.3-1747.0)$ & $2605.0(2518.0-2894.8)^{\mathrm{a}}$ & $1592.3(1467.8-1706.6)$ & $<0.001$ \\
Tissue weights $(\mathrm{g})$ & $0.23 \pm 0.03$ & $0.23 \pm 0.02$ & $0.23 \pm 0.03$ & 0.860
\end{tabular}

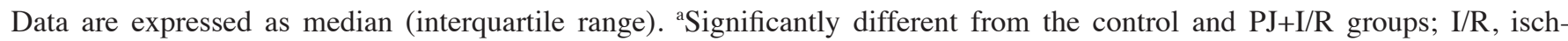
emia/reperfusion; PJ, pomegranate juice; t, tissue; s, serum; SOD, superoxide dismutase; MDA, malondialdehyde.

Table II. Comparison of the concentrations of spermatogenic cells between groups.

\begin{tabular}{lcccc}
\hline Cell type & Control & I/R & PJ+I/R & P-value \\
\hline Spermatids & $32.22(28.35-36.35)$ & $21.04(17.16-28.01)^{\mathrm{a}}$ & $28.68(24.86-37.24)$ & $\mathrm{P}<0.001$ \\
Spermatocytes & $18.56(15.53-21.27)$ & $11.53(8.89-14.57)^{\mathrm{a}}$ & $16.22(13.84-18.39)$ & $\mathrm{P}<0.001$ \\
Spermatogonia & $8.48(6.89-10.06)$ & $6.09(4.10-7.35)^{\mathrm{a}}$ & $7.81(6.41-9.28)$ & $\mathrm{P}=0.008$ \\
\hline
\end{tabular}

Data are expressed as median (interquartile range) $x 10^{6}$. ${ }^{a}$ Significantly different from the control and PJ+I/R groups; I/R, ischemia/reperfusion; PJ, pomegranate juice.

Finally, the spermatogenic cells in the seminiferous tubules, including the spermatids, spermatocytes and spermatogonia, were counted with an optical fractionator (Stereology workstation: Trinokulermicroskop Leica DM 2500, Leica Instruments; motorizedstage: Ludlelectronics, New York, NY, USA; digitalmicrocator: Heidenhain, Traunreut, Germany, StereoInvestigator: MBF Biosciences, Williston, VT, USA). The spermatogenic cell concentrations are expressed in number of cells/mm3).

Statistical analysis. Kruskal-Wallis one-way analysis of variance was used to compare the biochemical and sperm parameters among groups. For multiple comparisons, the Bonferroni adjusted Mann-Whitney U test was performed. Data are expressed as the median and interquartile range (IQR). $\mathrm{P}<0.05$ was considered to indicate a statistically significant difference. Analyses were carried out using a commercially-available software (IBM SPSS Statistics 19; IBM, Armonk, NY, USA).

\section{Results}

SOD activity and MDA levels in the serum and testicular tissue. The levels of MDA and SOD in the serum and tissue samples of the three groups of rats are presented in Table I. No statistically significant differences were identified between the groups in terms of testicular tissue weights. In the I/R group, the SOD activity and MDA levels in the serum and testicular tissue were significantly higher compared with those in the control group $(\mathrm{P}<0.001)$. The consumption of $\mathrm{PJ}$ significantly reduced the SOD activity and MDA levels in the serum and tissue compared with those in the I/R group $(\mathrm{P}<0.001)$.

Spermatogenic cell concentrations. The comparison of spermatogenic cell concentrations in the three groups of rats is presented in Table II. The concentrations of spermatids, spermatocytes and spermatogonia were significantly reduced in the I/R group compared with those in the control group $(\mathrm{P}<0.001)$. However, the consumption of PJ over a period of eight weeks significantly improved the spermatid, spermatocyte and spermatogonia concentrations compared with those in the $\mathrm{I} / \mathrm{R}$ group $(\mathrm{P}=0.008)$.

Histopathological analysis. In the histopathological analysis of the testicular tissues, intensive vacuolization with irregularities in the germ cell sequences were observed in the I/R group. A reduced incidence of vacuolizations and regular germ cell formation, comparable to that observed in the control group, were present in the $\mathrm{PJ}+\mathrm{I} / \mathrm{R}$ group. Representative microscopic images of the testicular tissues in the three groups are presented in Figs. 1-3.

\section{Discussion}

Since it contains a high amount of polyphenols, PJ possesses strong antioxidant properties. The antioxidant effects of polyphenols, particularly ellagitannins, have been previously 


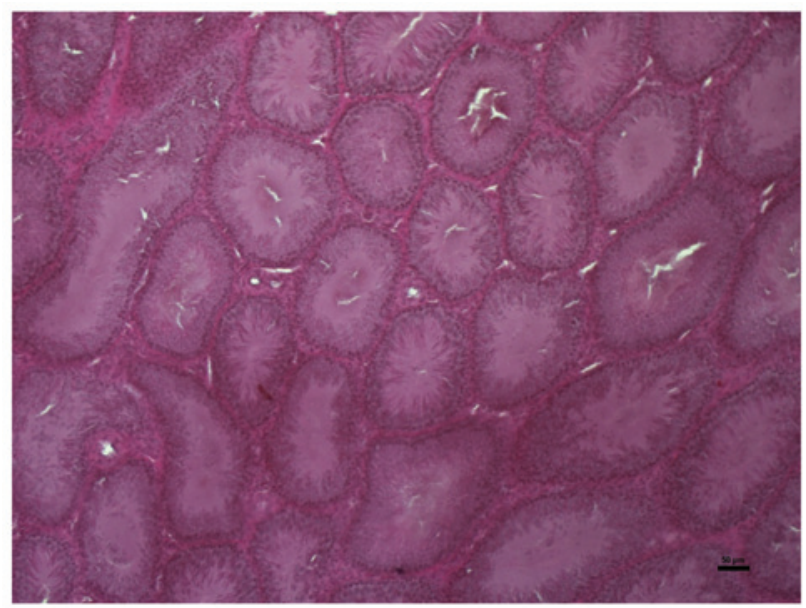

Figure 1. In the control group, the germ cells in seminiferous tubules were observed in regular form. Magnification, x10.

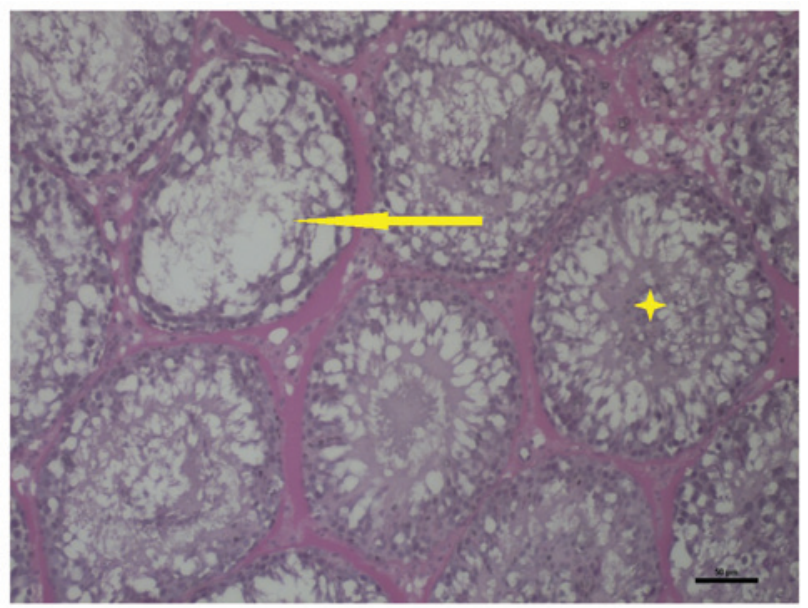

Figure 2. In the ischemia/reperfusion group, intensive vacuolization was observed in the seminiferous tubules (yellow arrow). Small numbers of spermatids, spermatocytes and spermatogonia were identified in the adluminal compartment, with irregularities in their germ cell sequences (yellow star). Magnification, x20.

demonstrated in numerous studies (17-19). Ellagitannins and other polyphenols are hydrolyzed into ellagic acid (EA), which is responsible for their antioxidant activities in vivo (20). An in vitro study has revealed the ability of EA to easily pass through the mitochondrial membrane (21). In the present study, testicular torsion-detorsion caused elevations in the levels of SOD and MDA, which are the indirect indicators of oxidative injury. However, pretreatment with PJ over eight weeks decreased the levels of SOD and MDA in the serum and testicular tissue.

Under normal conditions, sperm cells produce high amounts of ROS as a result of their physiological metabolism (22). As testicular tissue contains a high amount of unsaturated fatty acids, it is very sensitive to the detrimental effects of ROS. Lipid peroxidation is able to damage the structure of the lipid matrix in sperm membranes, decrease intracellular levels of ATP leading to reduced sperm viability, cause axonemal damage and increase mid-piece morphological defects (23. However, testicular tissue contains numerous

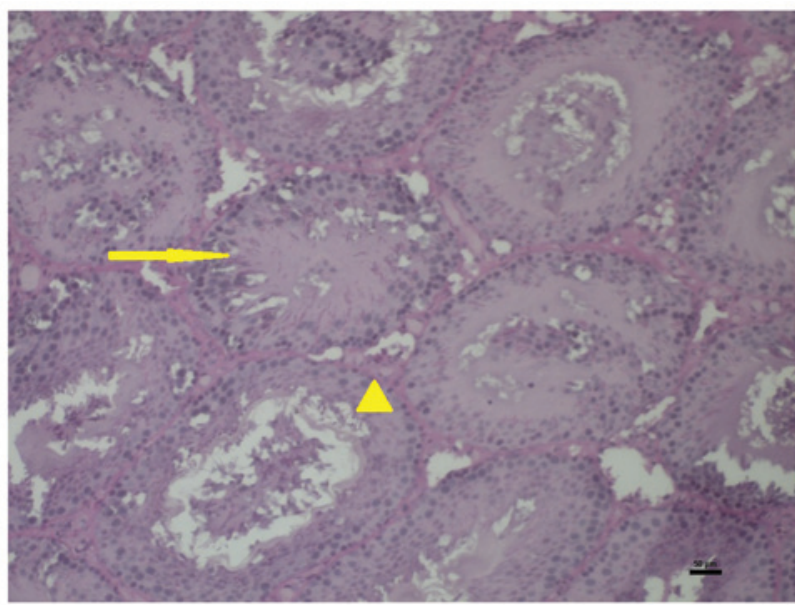

Figure 3. In the pomegranate juice + ischemia/reperfusion group, vacuolization and irregularities were observed in the seminiferous tubules (yellow arrowhead). However, similarly to the control group, a regular formation of germ cells was also detected in numerous seminiferous tubules (yellow arrow). Magnification, x20.

antioxidant enzymes and ROS scavengers that protect its spermatogenic function from OS and in particular from peroxidative damage, which is the most important cause of spermatogenic dysfunction $(24,25)$. This antioxidant defence system scavenges considerable numbers of ROS in order to protect sperm cells from their harmful effects and preserves only a small number of ROS to maintain normal cell function. Without this defence system, the ROS cause thinning of the germ layer, decreased spermatogenic cell density and sperm motility, and an increased production of abnormal sperm cells (26).

The current study demonstrated that testicular torsion-detorsion induced OS in testicular tissue and caused a reduction in the concentrations of all spermatogenic cell types, including spermatogonia, spermatocytes and spermatids. The reduction in spermatogenic cell concentration, which occurred without any reduction in testicular tissue weight, may indicate that OS was the most important reason for this pathology. However, the daily consumption of PJ over a period of eight weeks prior to the torsion-detorsion surgery resulted in a reduction in the OS parameters and a significant increase in the spermatogenic cell concentration.

Similarly, in a previous experimental study, Türk et al revealed that the daily consumption of PJ over a period of seven weeks caused a reduction in OS parameters and a marked increase in the levels of spermatogenic cells and the thickness of the germ layer (27). Furthermore, Mansour et al demonstrated that the daily consumption of PJ at various doses for a period of six weeks resulted in a significiant increase in serum glutathione peroxidase (GSH-Px) and catalase activities in rats. The study also noted that PJ consumption significantly increased epididymal sperm concentration and sperm motility parameters and decreased abnormal sperm production (28).

In the current experimental study, it was indicated that testicular torsion-detorsion caused OS, which may have led to reduced sperm concentrations in the rat testes. However, the daily consumption of PJ prior to the torsion-detorsion surgery 
resulted in a reduction in the parameters of OS and produced an increment in spermatogenic cell concentrations.

\section{References}

1. Aviram M, Dornfeld L, Kaplan M, Coleman R, Gaitini D, Nitecki et al. Pomegranate juice flavonoids inhibit low-density lipoprotein oxidation and cardiovasculardiseases: studies in atherosclerotic mice and in humans. Drugs Exp Clin Res 28 : 49-62, 2002

2. Seeram NP, Adams LS, Henning SM, Niu Y, Zhang Y, Nair MG and Heber D: In vitro antiproliferative, apoptotic and antioxidant activities of punicalagin, ellagic acid and a total pomegranate tannin extract are enhanced in combination with other polyphenols as found in pomegranate juice. J Nutr Biochem 16 : 360-367, 2005

3. Aslam MN, Lansky EP and Varani J: Pomegranate as a cosmeceutical source: pomegranate fractions promote proliferation and procollagen synthesis and inhibit matrix metalloproteinase-1 production in human skin cells. J Ethnopharmacol 103: 311-318, 2006.

4. Aviram M and Dornfeld L: Pomegranate juice consumption inhibits serum angiotensin converting enzyme activity and reduces systolic blood pressure. Atherosclerosis 158: 195-198, 2001.

5. Aviram M, Rosenblat M, Gaitini D, Nitecki S, Hoffman A, Dornfeld L, et al: Pomegranate juice consumption for 3 years by patients with carotid artery stenosis reduces common carotid intima-media thickness, blood pressure and LDL oxidation. Clin Nutr 23: 423-433, 2004

6. Seeram N, Lee R, Hardy M and Heber D: Rapid large scale purification of ellagitannins from pomegranate husk, a byproduct of the commercial juice industry. Sep Purif Technol 41: 49-55, 2005.

7. Hermann DD: Naturoceutical agents in the management of cardiovascular disease. Am J Cardiovasc Drugs 2: 173-196, 2002.

8. Seeram NP. Aviram M, Volkova N, Zhang Y, Henning SM, Nair $M$ and Heber D: Dietary polyphenols derived from pomegranates are potent antioxidants: evaluation in various in vitro models of antioxidation. Presented at 228th National Meeting of the American Chemical Society. (Abstract AGFD44), 2004 http://oasys2.confex.com/acs/228nm/techprogram/P787337.HTM

9. Gil MI, Tomás-Barberán FA, Hess-Pierce B, Holcroft DM and Kader AA: Antioxidant activity of pomegranate juice and its relationship with phenolic composition and processing. J Agric Food Chem 48: 4581-4589, 2000.

10. Turner TT, Bang HJ and Lysiak JL: The molecular pathology of experimental testicular torsion suggests adjunct therapy to surgical repair. J Urol 172: 2574-2578, 2004

11. Aitken RJ, Baker MA and Sawyer D: Oxidative stress in the male germ line and its role in the aetiology of male infertility and genetic disease. Reprod Biomed Online 7: 65-70, 2003.
12. Tremellen K: Oxidative stress and male infertility - a clinical perspective. Hum Reprod Update 14: 243-258, 2008

13. Sanocka D and Kurpisz M: Reactive oxygen species and sperm cells. Reprod Biol Endocrinol 2: 12, 2004.

14. de Lamirande E, Jiang H, Zini A, Kodama H and Gagnon C: Reactive oxygen species and sperm physiology. Rev Reprod 2 48-54, 1997.

15. Sun Y, Oberley LW and Li Y: A simple method for clinical assay of superoxide dismutase. Clin Chem 34: 497-500, 1988.

16. Esterbauer $\mathrm{H}$ and Cheeseman KH: Determination of aldehydic lipid peroxidation products: malonaldehyde and 4-hydroxynonenal. Methods Enzymol 186: 407-421, 1990.

17. Manach C, Mazur A and Scalbert A: Polyphenols and prevention of cardiovascular diseases. Curr Opin Lipidol 16: 77-84, 2005.

18. Cerdá B, Espín JC, Parra S, Martínez P and Tomás-Barberán FA. The potent in vitro antioxidant ellagitannins from pomegranate juice are metabolised into bioavailable but poor antioxidant hydroxy-6H-dibenzopyran-6-one derivatives by the colonic microflora of healthy humans. Eur J Nutr 43: 205-220, 2004.

19. Cerdá B, Llorach R, Cerón JJ, Espín JC and Tomás-Barberán FA. Evaluation of the bioavailability and metabolism in the rat of punicalagin, an antioxidant polyphenol from pomegranate juice. Eur J Nutr 42: 18-28, 2003

20. Larrosa M, Tomás-Barberán FA and Espín JC: The dietary hydrolysable tannin punicalagin releases ellagic acid that induces apoptosis in human colon adenocarcinoma Caco- 2 cells by using the mitochondrial pathway. J Nutr Biochem 17: 611-625, 2006.

21. Seeram NP, Lee R and Heber D: Bioavailability of ellagic acid in human plasma after consumption of ellagitannins from pomegranate (Punica granatum L.) juice. Clin Chim Acta 348: 63-68, 2004.

22. Sikka SC: Oxidative stresss and role of antioxidants in normal and abnormal sperm function. Front Biosci 1: e78-e86, 1996.

23. Henkel R: The impact of oxidants on sperm functions. Andrologia 37: 205-206, 2005.

24. Koksal IT, Usta M, Orhan I, Abbasoglu S and Kadioglu A: Potential role of reactive oxygen species on testicular pathology associated with infertility. Asian J Androl 5: 95-99, 2003

25. Turner TT and Lysiak JJ: Oxidative stress: a common factor in testicular dysfunction. J Androl 29: 488-498, 2008.

26. Alkan I, Simşek F, Haklar G, Kervancioğlu E, Ozveri H, Yalçin S and Akdaş A: Reactive oxygen species production by the spermatozoa of patients with idiopathic infertility: relationship to seminal plasma antioxidants. J Urol 157: 140-143, 1997.

27. Türk G, Sönmez M, Aydin M, Yüce A, Gür S, Yüksel M, Aksu EH and Aksoy H: Effects of pomegranate juice consumption on sperm quality, spermatogenic cell density, antioxidant activity and testosterone level in male rats. Clin Nutr 27: 289-96, 2008.

28. Mansour SW, Sangi S, Harsha S, Khaleel MA, Ibrahim AR and Eldin TA: Sensibility of male rats fertility against olive oil, Nigella sativa oil and pomegranate extract. Asian Pac J Trop Biomed 3: 563-568, 2013. 